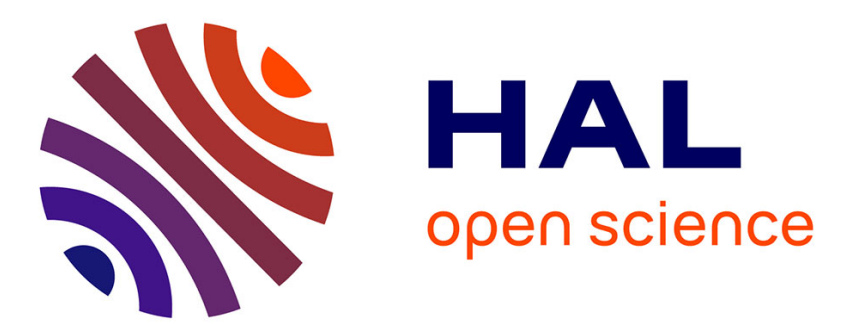

\title{
Highly ordered biobased scaffolds: From liquid to solid foams
}

\author{
Sébastien Andrieux, Wiebke Drenckhan, Cosima Stubenrauch
}

\section{To cite this version:}

Sébastien Andrieux, Wiebke Drenckhan, Cosima Stubenrauch. Highly ordered biobased scaffolds: From liquid to solid foams. Polymer, 2017, 126, pp.425-431. 10.1016/j.polymer.2017.04.031 . hal03528705

\section{HAL Id: hal-03528705 \\ https://hal.science/hal-03528705}

Submitted on 17 Jan 2022

HAL is a multi-disciplinary open access archive for the deposit and dissemination of scientific research documents, whether they are published or not. The documents may come from teaching and research institutions in France or abroad, or from public or private research centers.
L'archive ouverte pluridisciplinaire HAL, est destinée au dépôt et à la diffusion de documents scientifiques de niveau recherche, publiés ou non, émanant des établissements d'enseignement et de recherche français ou étrangers, des laboratoires publics ou privés. 


\title{
Highly Ordered Biobased Scaffolds: From Liquid to Solid Foams
}

\author{
Sébastien Andrieux ${ }^{1}$, Wiebke Drenckhan ${ }^{2}$, Cosima Stubenrauch $^{1 *}$ \\ ${ }^{1}$ Institute of Physical Chemistry, University of Stuttgart, Stuttgart, Germany \\ ${ }^{2}$ Laboratoire de Physique des Solides, Université Paris-Sud, Orsay, France
}

\begin{abstract}
We propose here a foam templating route using microfluidic techniques to produce biobased monodisperse chitosan foams from aqueous chitosan solutions which are stabilized with a biobased sugar surfactant. The foaming solution contained $1.5 \mathrm{wt} \%$ chitosan and was stabilized by $0.1 \mathrm{wt} \%$ surfactant. We show how microfluidics allows for the generation of a wide range of bubble sizes, from $200 \mu \mathrm{m}$ to $800 \mu \mathrm{m}$, with the help of microfluidic chips with constrictions of different sizes. Due to the monodispersity of the resulting bubbles, highly ordered liquid foams are formed. We freeze-dried the non-cross-linked chitosan liquid foams to obtain solid chitosan foams. In addition, we also cross-linked the liquid templates by genipin, a natural cross-linker, via heating at $40{ }^{\circ} \mathrm{C}$ for $2 \mathrm{~h}$. The aqueous cross-linked foams were dried, either by heating at $60{ }^{\circ} \mathrm{C}$ for $18 \mathrm{~h}$ or by freeze-drying. We found that the drying method, as well as whether the foams were cross-linked or not, have a tremendous influence on the morphology of the solid foams.
\end{abstract}

*Corresponding author 


\section{Introduction}

Polymer foams or macroporous polymers are materials composed of gas bubbles embedded in a polymer matrix. Such materials are characterized by a low weight and a high specific surface area, making them valuable materials for numerous applications. The structural and mechanical properties of solid foams are mainly dictated by their morphology, i.e. the size of the pores, the size of the openings between the pores (if any), the thickness of the walls, the fine structure of the walls, and the relative density. Although the size of the pores does not significantly affect the mechanical properties of the material for a given relative density, it is an important parameter for biological applications where solid foams are used as scaffolds for tissue engineering. Cell colonization calls for monodisperse pores with sizes between $100 \mu \mathrm{m}$ and $500 \mu \mathrm{m}[1]$. The foams also have to be open-cell in order to allow the transportation of nutrients and oxygen throughout the scaffold. Well-defined scaffolds are required for systematic studies as local differences of the structure affect cell growth and result in different biodegradation rates of the scaffolds [2-4]. Moreover, the optimal pore size differs for each type of cell to be cultivated which is why we need methods allowing an easy and straightforward tailoring of the scaffold's pore size.

High Internal Phase Emulsions (HIPEs) have been proposed as templates for porous polymers (thus named polyHIPEs) with an improved control over their structures [5]. However, typical polyHIPEs are materials with still too large pore size distributions and pore sizes below 100 $\mu \mathrm{m}$. It was only recently that both the control of polydispersity and of the pore size were tackled by using microfluidic techniques, which allowed for the production of pores with diameters in the range of $25-250 \mu \mathrm{m}$ [4]. But a major problem remains, specific to the use of emulsions: the need for an organic solvent. Whether it is used as the dispersed phase itself or to wash the dispersed phase out of the material, traces of solvent in scaffolds are always undesirable. Foam templating, on the contrary, uses gas as the dispersed phase, which solves the problem of the need for an organic solvent [2]. Thus, we use foam templating combined with microfluidics to reach a high structural order and to tune the bubble sizes [3,6-9].

Foam templating, like any other type of colloidal templating, is based on the idea that the liquid template can be solidified in order to produce a solid material that is, ideally, the exact image of its liquid counterpart. The aim of templating is to tailor the pore size, the pore size distribution and the pore morphology of the solid foam by tailoring the structure of the liquid template. We are thus faced with two challenges. The first challenge is to produce a liquid 
template with the desired structure, which will be addressed in Section 3.1. The second challenge inherent to foam templating is to retain the structure of the liquid template throughout solidification. We will largely discuss this issue in Sections 3.2 and 3.3.

The polymer used in the study at hand is chitosan, which is a polysaccharide derived from chitin. Chitin is extracted from the shells of crustaceans [10]. Chitosan is slightly soluble in acidic solutions, in which its amino groups are protonated. Chitosan is thus a polycation and displays the common properties of polyelectrolytes [11]. Chitosan has already been studied extensively as a potential material for tissue engineering and drug release [12,13]. Indeed, thanks to its amino side groups, chitosan is intrinsically antibacterial. Chitosan also displays little reaction with foreign bodies and can be degraded via enzymatic activity into oligosaccharides [12].

Aqueous chitosan solutions can form hydrogels if the chitosan is cross-linked. Such hydrogels have been extensively studied on their own, focusing on the kinetics of cross-linking, the swelling behavior, or on the use as biomaterials $[14,15]$. Miras et al. developed an emulsion templating route to produce porous cross-linked chitosan using genipin as cross-linker [16]. They obtained pores with diameters of $\sim 0.5 \mu \mathrm{m}$ from sheared emulsions whose droplets had a diameter of $\sim 1.5 \mu \mathrm{m}$. The dispersed phase of the emulsion template was decane and the emulsion was stabilized by an ethoxylated alcohol (Synperonic A7). The mechanical properties of the resulting chitosan foams were not characterized. Testouri et al. [8] proposed a foam templating route via microfluidics to produce monodisperse cross-linked chitosan foams. The resulting chitosan foams were monodisperse and had bubble sizes between 1 and $3 \mathrm{~mm}$. However, the surfactant used was not biobased and used in huge quantities (1000 times the critical micellar concentration). Furthermore, chitosan was cross-linked with glyoxal, which is a dialdehyde that is neither biobased nor adapted for biomedical applications. Moreover, the pore size was not varied, nor were the morphologies of the solid foams, which were dried at room temperature.

We propose here a foam templating route using microfluidic techniques to produce monodisperse chitosan foams which are stabilized with a biobased sugar surfactant and then cross-linked with genipin, which is also biobased. We show how microfluidics allows for the generation of a wide range of bubble sizes, namely from $200 \mu \mathrm{m}$ to $800 \mu \mathrm{m}$. Highly ordered liquid foams form due to the monodispersity of the bubbles. We followed several routes in order to obtain solid foams from liquid templates, which are summarized in Figure 1. We were 
able to directly freeze-dry the non-cross-linked liquid foams (route 1) into solid foams. In addition, we also cross-linked the liquid foams by heating them to $40{ }^{\circ} \mathrm{C}$ (route 2). Once crosslinked, the aqueous foams (which are now foamed hydrogels) were dried, either via freezedrying (route $3 \mathrm{a}$ ) or via heating to $60^{\circ} \mathrm{C}$ (route $3 \mathrm{~b}$ ).

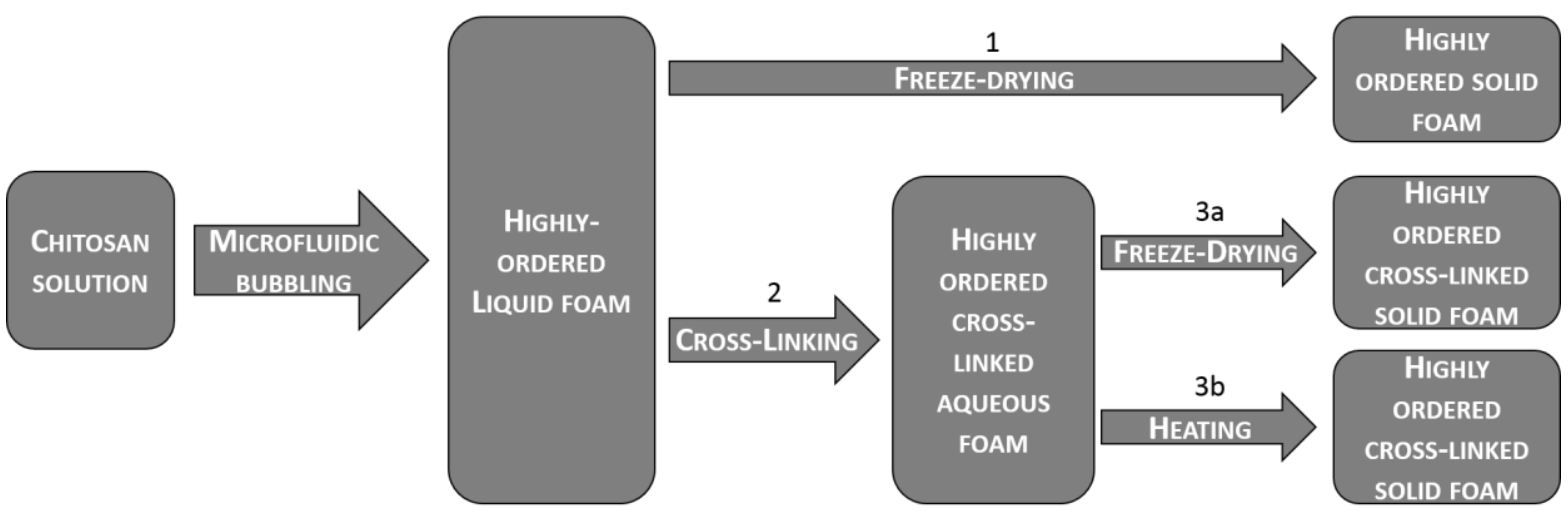

Figure 1: Different routes to highly ordered chitosan scaffolds with various morphologies.

\section{Experimental Section}

\section{Chemicals}

Chitosan was purchased from Sigma-Aldrich and has a molecular weight of $300,000 \mathrm{~g} \mathrm{~mol}^{-1}$ and a deacetylation degree of ca. $80 \%$ (data from supplier). Glacial acetic acid was purchased from VWR. The water used was bidistilled. The surfactant, Plantacare 2000 UP, was donated by Cognis Deutschland GmbH \& Co (now BASF). Plantacare 200 UP is an alkyl polyglycoside with a head group composed of 1.5 glycoside units and alkyl chains between 8 and 16 carbons. The cross-linker genipin was purchased from Challenge Bioproducts Co., LTD. Perfluorohexane was purchased from Alfa Aesar. The polymer used to make the microfluidic chip was COC (Cyclic Olefin Copolymer), and was kindly donated by TOPAS Advance Polymers. All chemicals were used without further purification.

\section{Preparation of Chitosan Solutions}

Chitosan was dissolved in a solution containing $0.2 \mathrm{~mol} \mathrm{~L}^{-1}$ acetic acid and $0.05 \mathrm{~mol} \mathrm{~L}^{-1}$ sodium acetate with a paddle stirrer at room temperature overnight. The initial chitosan concentration was $1.5 \mathrm{wt} \%$, while the final chitosan concentration is a bit lower since impurities were removed by filtration under vacuum using filter paper (\#113 from Whatman, with a pore size of $30 \mu \mathrm{m})$. The surfactant was added such that a concentration of $0.1 \mathrm{wt} \%$ was obtained, which 
corresponds to $10 \mathrm{cmc}$. A concentration of $10 \mathrm{cmc}$ is sufficient to provide a good stability of the foam but is low enough for the surfactant not to act as co-solvent. However, no foam stability study was conducted for different surfactant concentrations, which is why we cannot say whether a lower / higher surfactant concentration would have an impact on the results. Genipin was added directly to the chitosan solution which was stirred until the genipin crystals were completely dissolved. The concentration of genipin was $0.2 \mathrm{wt} \%$. The dissolution of genipin was carried out in an ice bath to exclude cross-linking before foam formation.

\section{Microfluidic Device}

Microfluidic bubbling was carried out using two different chips, namely a home-made plastic chip and a commercial glass chip. Although chips of different materials are used we did not encounter dewetting problems. The foams are produced at low gas volume fractions, i.e. that the bubbles are surrounded by enough liquid and do not adhere to the hydrophobic channel wall. The plastic chip was made by micromilling and molding according to the process described in [8]. The plastic chip has a flow-focusing geometry, with a constriction of square section with a height and width of $400 \mu \mathrm{m}$. We denote this chip as the "400 $\mu \mathrm{m}$ chip" throughout this paper. The glass chip was purchased from Dolomite Microfluidics and its Xjunction has a depth of $190 \mu \mathrm{m}$ and a width of $195 \mu \mathrm{m}$. We denote this chip as the "190 $\mu \mathrm{m}$ chip" throughout this paper. Both chips are shown in Figure S1 with the dimensions of the channels. The flows were pressure-driven using an OB1 Mk2 Pressure Controller from Elveflow connected to a nitrogen tap. The gas phase was nitrogen with traces of perfluorohexane in order to hinder Ostwald ripening. A small amount of liquid perfluorohexane was put in a glass bottle sealed with a GL45 cap from Vaplock allowing the plugging of the tubings from the pump and to the chip. Traces of perfluorohexane were carried away with the flowing nitrogen into the chip. Similarly, the chitosan solution was kept in a sealed bottle plugged between the chip and the pressure pump, allowing the liquid to be pushed into the chip by the pressure applied by the pump into the bottle. The bottle containing the chitosan solution was kept in an ice bath at all times to prevent premature cross-linking. Bubbling was followed by means of a Nikon SMZ-800N optical microscope coupled with an Optronis CL600X2 highspeed camera. The foams were collected in polystyrene Petri dishes. The foams were $1 \mathrm{~mm}$ high and had a diameter of $5.5 \mathrm{~cm}$. The time required to collect a foam depended strongly on the liquid pressure and ranged from $1 \mathrm{~min}$ to $20 \mathrm{~min}$. 


\section{Foam Cross-linking}

The cross-linking method consisted in placing the foams right after their formation in an oven at $40{ }^{\circ} \mathrm{C}$ for two hours before storage at room temperature.

\section{Foam Drying}

The foams, cross-linked or not, were stored at $-60{ }^{\circ} \mathrm{C}$ before freeze-drying. The samples were then frozen in liquid nitrogen and subsequently freeze-dried in an Alpha 2-4 LDplus freezedrier from CHRIST. An alternative drying process was to place the foams in an oven at $60{ }^{\circ} \mathrm{C}$ for 18 hours. $60{ }^{\circ} \mathrm{C}$ is a temperature high enough for a quick evaporation but not high enough to "boil" the foam and destroy the foam's structure. The 18 hours are not optimised and fixed by convenience with drying the samples over night. To verify that the foams were dry, their weight was determined after the 18 hours before they were put back into the oven for another two hours at $60^{\circ} \mathrm{C}$, and weighed again. Since there was no additional weight loss after this extra drying time, all samples were assumed to be dry after $18 \mathrm{~h}$.

\section{Bubble Size and Pore Size Distribution}

The bubble size distributions were determined using the image analysis software ImageJ from microscopy images taken with the Nikon SMZ-800N optical microscope coupled with the Optronis CL600X2 high-speed camera. Note that the bubble/pore sizes discussed throughout this work are characteristic diameters and not radii. The bubble and pore sizes were determined by measuring the center-to-center distance between two bubbles/pores. The bubble/pore center was defined as the average position of all pixels belonging to the bubble/pore. Note that neighbouring bubbles touch and are in close contact with a characteristic separation of the order of $100-1000 \mathrm{~nm}$ at the contact points. The black rings which are visible in Figure 3 (a) and (d) are not the limits of the liquid bubbles, but are an optical effect [17]. At least 60 bubbles were measured for each sample to determine the polydispersity index (PDI). The PDI was used to assess the monodispersity of the liquid and solid foams and reads

$$
P D I=100 \cdot \frac{\sqrt{\left\langle d^{2}\right\rangle-\langle d\rangle^{2}}}{\langle d\rangle} .
$$

A sample is called monodisperse if its $P D I$ is $<5 \%$ [18]. The structure of the solid foams was investigated with scanning electron microscopy (SEM) using a CamScan CS 44 microscope at a voltage of $5 \mathrm{kV}$. The solid foams were dipped into liquid nitrogen before being cut with a 
scalpel into samples small enough for SEM. Without this freezing step, the foams were not stiff enough to make clear cuts and tore upon cutting.

\section{Results and Discussion}

\subsection{Generation of Monodisperse Liquid Foams}

In Figure 2 one sees the range of bubble sizes which can be generated with the $190 \mu \mathrm{m}$ and the $400 \mu \mathrm{m}$ chip, respectively. Bubbles from $180 \mu \mathrm{m}$ up to $850 \mu \mathrm{m}$ diameter can be formed. Since the bubble size depends on the size of the constriction [19], the $190 \mu \mathrm{m}$ chip allows the formation of the smaller bubbles, while the $400 \mu \mathrm{m}$ chip can be used for the larger bubbles. In this particular case, the range of bubble sizes is $\sim 165-365 \mu \mathrm{m}$ for the $190 \mu \mathrm{m}$ chip and $\sim 370$ - $845 \mu \mathrm{m}$ for the $400 \mu \mathrm{m}$, which complements each other perfectly. Note that the standard deviations of the bubble sizes are larger for the $400 \mu \mathrm{m}$ chip, yielding foams with a higher PDI. We assign this effect to the larger dimensions of the $400 \mu \mathrm{m}$ chip. Since the channels and tubings are larger, the pressures required to push the liquid are lower than for the $190 \mu \mathrm{m}$ chip. Unfortunately, lower pressures result in a more fluctuating pressure controller and these fluctuations slightly decrease the monodispersity of the liquid foam. Although only one liquid pressure is shown for each chip, the liquid pressure can be varied as easily as the gas pressure. Note that the bubble size is mainly dictated by the pressure ratio provided that the bubbling regime remains the same $[19,20]$.

Although microbubbling allows for the explicit tuning of the liquid and gas flow rates and thus of the liquid fraction in the microfluidic channels, the liquid foam collected in the petridish is subject to gravity-driven drainage, which is evidenced by an excess liquid phase. In our case, the low liquid fraction of the final foams thus is not set by the liquid and gas flow rates or the chip material, but is dictated by gravity [21]. 


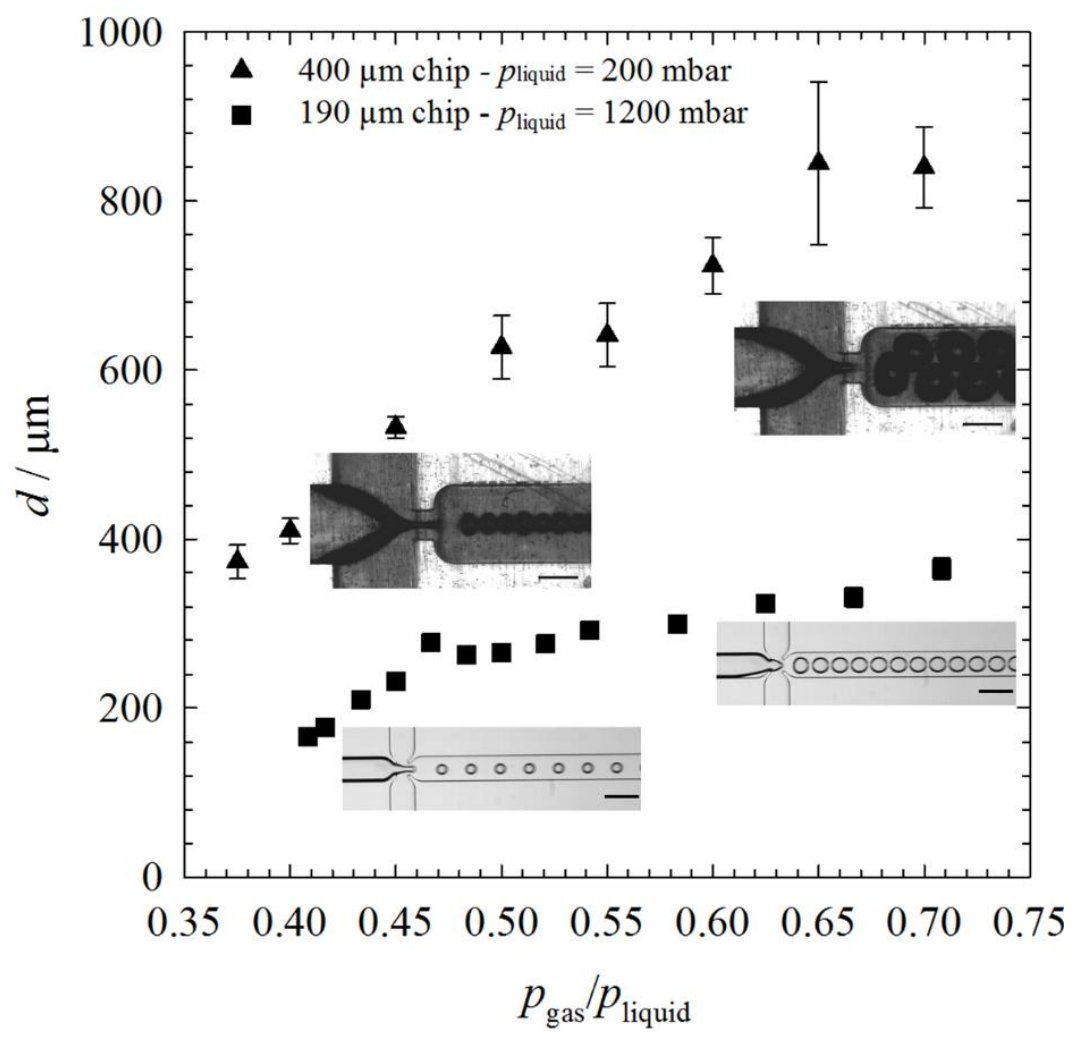

Figure 2: Bubble diameter $d$ plotted versus the ratio of gas and liquid pressure $p_{\text {gas }} / p_{\text {liquid }}$ for the $190 \mu \mathrm{m}$ and the $400 \mu \mathrm{m}$ microfluidic chips. The error bars represent the standard deviation of the bubble sizes of the collected foams and are smaller than the symbols for the $190 \mu \mathrm{m}$ chip. The photographs are microscopic images of the bubble formation. The bubbles are formed at gas pressures of $75 \mathrm{mbar}$ and $110 \mathrm{mbar}$ for the $400 \mu \mathrm{m}$ chip, and $520 \mathrm{mbar}$ and $800 \mathrm{mbar}$ for the $190 \mu \mathrm{m}$ chip. The scale bars are $500 \mu \mathrm{m}$.

\subsection{From Liquid to Solid Foams}

The bubbles generated with the microfluidic device were collected in Petri dishes and were left under atmospheric conditions until an acceptable quantity of foam was produced. Self-ordering does not require specific experimental conditions as long as the foam is monodisperse enough. In Figures 3 (a) and (d) one sees examples of monodisperse ordered liquid foams produced by both microfluidic chips. The average bubble sizes are $338 \pm 8 \mu \mathrm{m}$ and $644 \pm 30 \mu \mathrm{m}$ for the foams prepared with the $190 \mu \mathrm{m}$ chip and the $400 \mu \mathrm{m}$ chip, respectively. The foam prepared with the $190 \mu \mathrm{m}$ chip has a PDI of $2.4 \%$, while the one prepared with the $400 \mu \mathrm{m}$ chip has a PDI of $4.7 \%$. Since both PDIs are below 5\%, both liquid foams are to be considered as monodisperse [18]. Looking at the liquid foam templates in Figure 3 (a) and (b) one nicely sees the crystalline arrangements of the bubbles. 
For foam templating to work, the liquid template needs to remain stable within the time frame of solidification. To remove this constraint, we froze the foams in a freezer to $-60{ }^{\circ} \mathrm{C}$ directly after their formation, so that neither coalescence nor coarsening can occur. Despite the presence of genipin in the chitosan solution, cross-linking cannot occur at such a low temperature, as the genipin molecules are trapped in the ice crystals. The absence of cross-linking can be verified by the absence of blue color in the foam, as the reaction of genipin with amino groups in presence of oxygen causes the system to become blue. In other words, following the time evolution of the blue color allows a macroscopic monitoring of the cross-linking reaction $[14,22,23]$.

The foams were then placed in liquid nitrogen and freeze-dried, which resulted in noncrosslinked, solid monodisperse foams such as the ones shown in Figure 3 (b) and (e). The pores of all solid foams are smaller than the corresponding bubbles, indicating a shrinkage, which is typical of freeze-dried materials. However, shrinkage is not the only phenomenon to account for the smaller pore sizes. When cutting a solid foam, we do not cut the pores in their hemispheres. As a result, the pore sizes measured via microscopy are underestimated and the PDI is overestimated.

One can see in Figure 3 (b) the ordering of the pores in a plane of the cut, while such ordering is not visible for the material produced with the $400 \mu \mathrm{m}$ chip shown in Figure 3 e). One reason is the fact the that cut is not as nice as the one for the foam in Figure 3 (b) and one actually observes several planes at the same time. Another reason is the observation that the $400 \mu \mathrm{m}$ chip tends to yield foams with a higher PDI. Since monodispersity is necessary for selfordering, an increase in PDI may result in more structural defects. However, all scaffolds showed hexagonally ordered pores on their top layer before the samples were cut (see Figure S2 in the supplementary information). The non-cross-linked chitosan scaffolds are soft and recover their initial shape after deformation. The chitosan scaffolds need to be frozen into liquid nitrogen to become hard enough to allow clean cuts. The chitosan scaffolds are open-cell foams and their windows (or interconnections) have an average diameter of $63 \pm 12 \mu \mathrm{m}$ and $67 \pm 15$ $\mu \mathrm{m}$ for the $190 \mu \mathrm{m}$ chip generated scaffold and the $400 \mu \mathrm{m}$ chip generated scaffold, respectively. Note the presence of thick rings surrounding some windows, which indicates that the films do not solely contain surfactant but also chitosan when they break. 

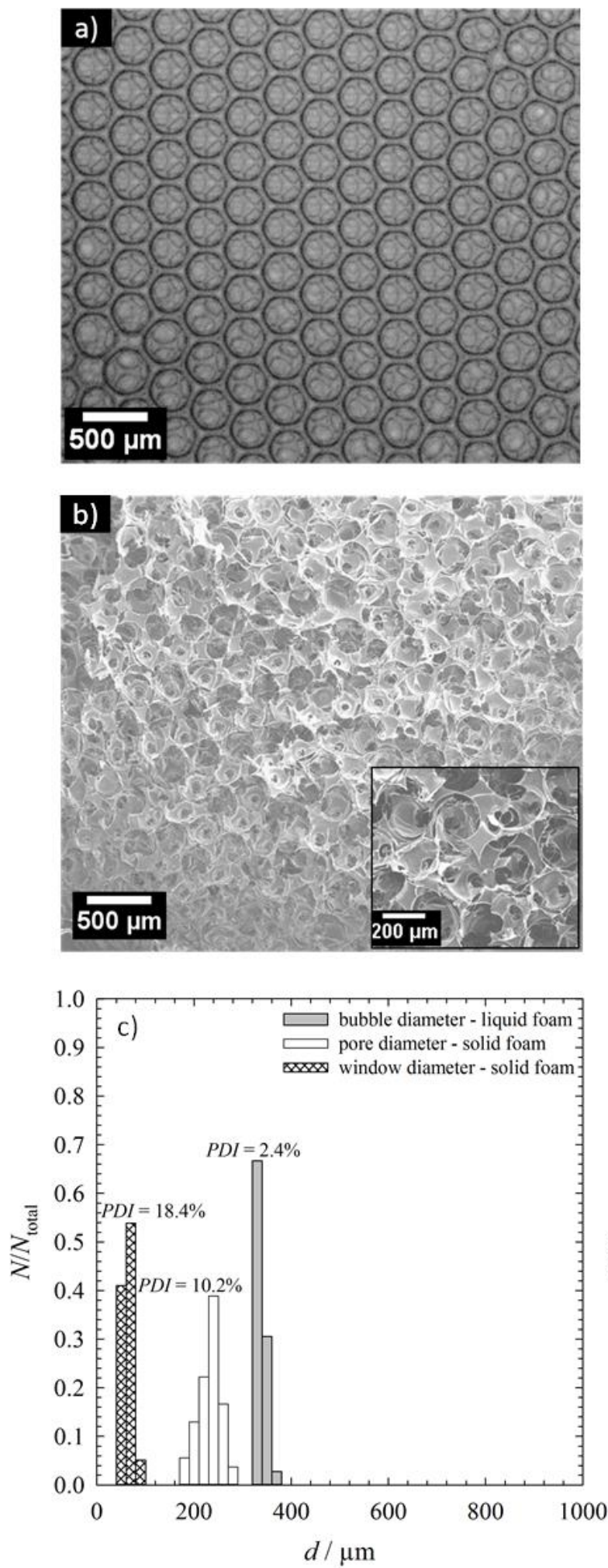
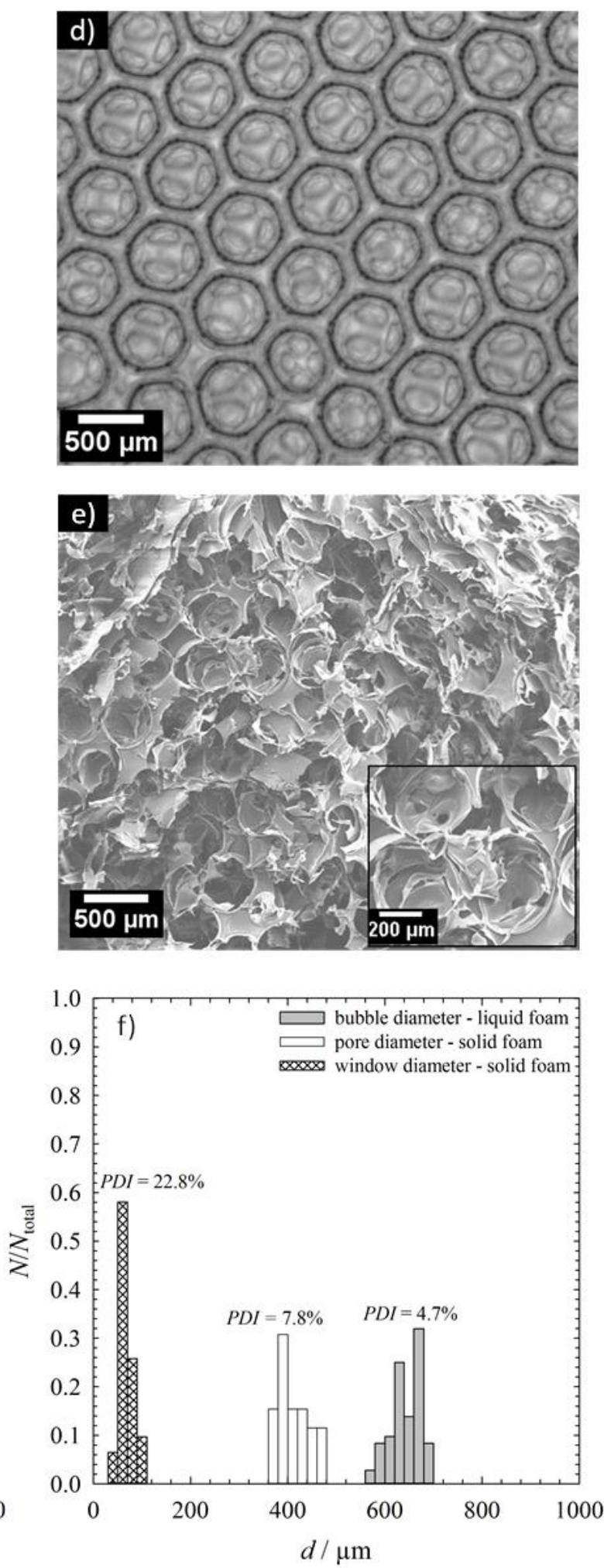

Figure 3: Pictures of monodisperse foams prepared with (a) the $190 \mu \mathrm{m}$ chip and (d) the $400 \mu \mathrm{m}$ chip. SEM pictures of the corresponding solid foams prepared by freeze-drying of the liquid foams directly after their formation: (b) was generated from (a) and (e) was generated from (d). The bubble, pore and window size distributions with PDIs shown in (c) were calculated from (a) and (b), while those shown in (f) were calculated from (d) and (e). 


\subsection{From Liquid to Cross-linked to Dried Foams}

All cross-linking experiments were carried out with foams produced with the $400 \mu \mathrm{m}$ chip. We have shown that freezing the liquid foams right after their formation and before freeze-drying stabilizes them. Moreover, freezing the foams prevents their cross-linking and the resulting solid foams are not cross-linked. In order to improve the mechanical properties and to obtain a solid foam that is not soluble in water, we thus need to cross-link the foams before drying them, which raises the challenge of foam stability during cross-linking. Two approaches are possible in order to reach sufficient foam stability within the time of cross-linking assuming that the cross-linked system has reached a storage modulus high enough to counteract coarsening and coalescence [24]. We can either decrease the cross-linking time or improve foam stability. Let us start with the former and see how we can shorten the cross-linking time.

A genipin molecule reacts with two amino groups of chitosan on two sites to form a cross-link $[14,15,22]$. One can follow this cross-linking reaction using oscillatory rheology and determine the gel point, which is defined as the intersection of the loss modulus and storage modulus as shown in the Figure S3. We recorded the gel point of the chitosan-genipin system at different temperatures and report the gel point dependency on the temperature in Figure S3. Increasing the temperature accelerates the cross-linking reaction in a controlled manner. One can thus reach the gel point after shorter times by heating the foam. Heating a liquid foam to $40{ }^{\circ} \mathrm{C}$ for two hours ensures cross-linking, which we verify via the dark blue color of the sample [14] and its inability to flow. Looking at Figures 4 (a) and (b) one sees that cross-linking occurred while retaining the order of the bubbles as well as the monodispersity, with PDIs of $2.9 \%$ for the liquid foam and $3.3 \%$ for its cross-linked counterpart. The cross-linked foam shown in Figure 4 (b) has various crystalline structures, such as hcp (hexagonal close-packed, on the left part of the picture), fcc(100) (face-centered cubic, on the upper right of the picture) and fcc(111) (on the lower right of the picture) [25]. The corresponding bubble size distributions in Figure 4 (d) show that cross-linking does not significantly affect the bubble size distribution or the PDI. Moreover, it shows that foam ageing mechanisms such as coarsening or coalescence are avoided thanks to cross-linking. If the structure of the foam is retained through cross-linking, water can be removed via simple heating. As shown in Figure 4 (c), one can produce a crosslinked solid foam by heating a cross-linked aqueous foam to $60{ }^{\circ} \mathrm{C}$ in an oven for $18 \mathrm{~h}$. The obtained material is hard and brittle. The PDI of the dried cross-linked foam, equal to $3.6 \%$, confirms monodispersity. Furthermore, the pore size distribution overlaps well the bubble sizes distributions of the non-cross-linked and the cross-linked aqueous foams. The resulting solid 
foam has polyhedral structures with crystalline regions separated by joints and defects. Note that the foams have well-defined holes in the films, i.e. they are open-cell foams. A close look at the inset of Figure 4 (c) reveals hole boundaries. To guide the reader's eye we indicated two regions of the foam where the film has not ruptured and thus is greyer compared to the regions where the films have ruptured (the darker the colour, the more material). The thin struts akin to Plateau borders of a low density foam suggest that, despite cross-linking, the chitosan molecules reorganized themselves through drying as a response to the evaporation of the solvent. Although interesting, this structure had collapsed into a quasi-2D structure and the different layers just sit on top of each other. We attribute this collapse to the fact that the foam does not have sufficient strength to carry its own weight once the films rupture during the evaporation of water. The inner pressure of the bubbles can thus no longer prevent the foam from collapsing. In order to avoid this effect, the elastic modulus of the dried Plateau borders need to be high enough so that the overall elastic modulus of the foam is sufficiently high to carry its own weight. This could be done by working with higher chitosan concentrations, stronger cross-linking or additives. 

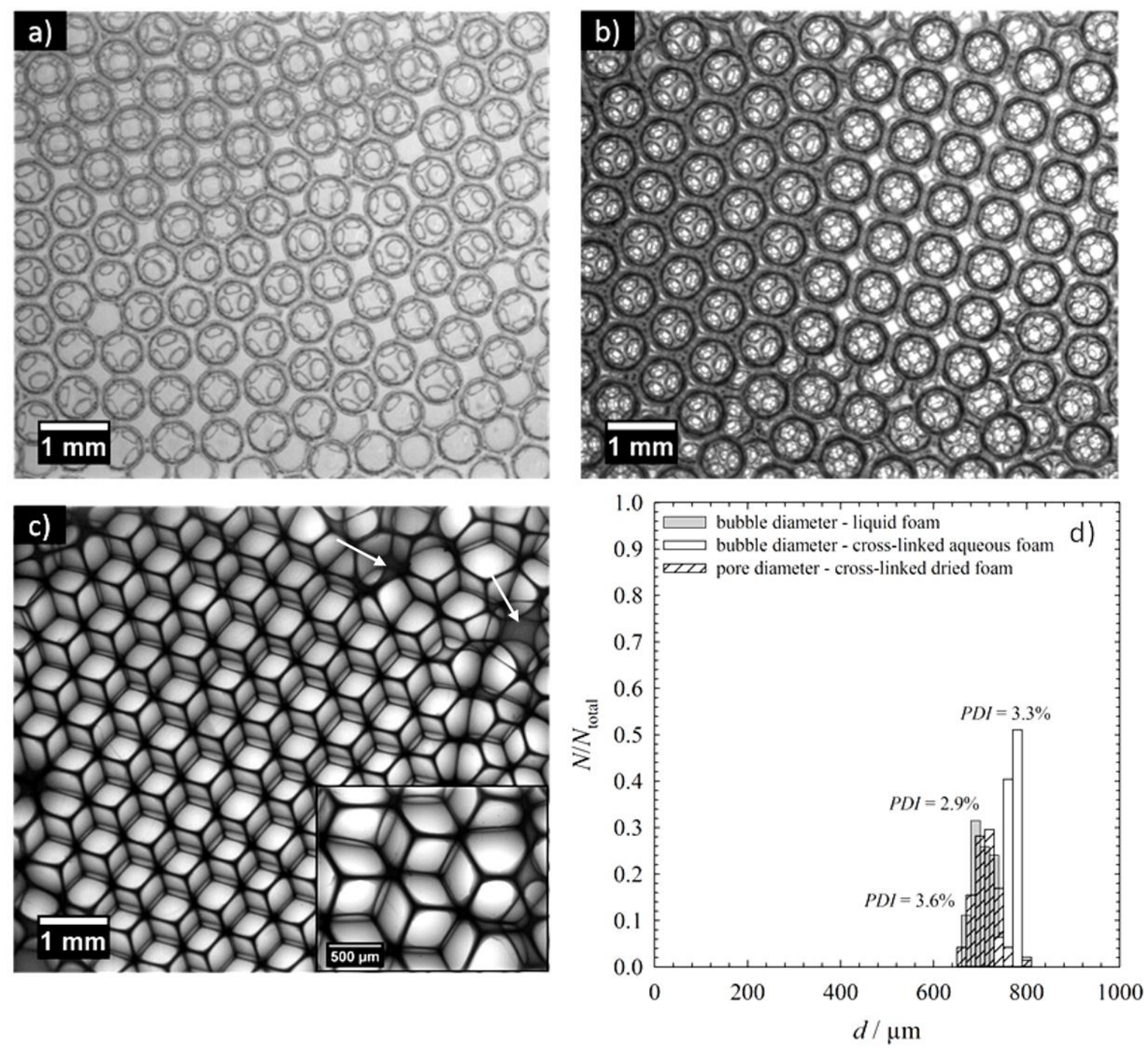

Figure 4: Pictures of chitosan foams (a) right after microfluidic bubbling, (b) after $2 \mathrm{~h}$ at $40{ }^{\circ} \mathrm{C}$ (cross-linking) and then $18 \mathrm{~h}$ at room temperature, (c) after $2 \mathrm{~h}$ at $40{ }^{\circ} \mathrm{C}$ (cross-linking) and then $18 \mathrm{~h}$ at $60{ }^{\circ} \mathrm{C}$ (drying). (d) Bubble and pore size distributions of the different foams with their corresponding PDIs. The white arrows in (c) point to films that have not ruptured during solidification

We have seen that freeze-drying non-cross-linked foams renders bulk solid foams while drying via heating leads to the collapse of the foams, no matter whether cross-linked or not. Since cross-linking does not affect the structure of the liquid templates, we freeze-dried cross-linked foams to observe the influence of drying and cross-linking on the structure of the solid foams. Figure 5 (a) shows a picture of a freeze-dried cross-linked foam while Figure 5 (b) depicts its pore size distribution and the bubble size distribution of the corresponding liquid foam. Let us first look at the foam's structure, which strongly differs from that of non-cross-linked foams. Although freeze-dried non-cross-linked foams have spherical pores with windows, the freeze- 
dried cross-linked has a much more open morphology with only Plateau borders and no walls. The dried Plateau borders, which have the morphology of Plateau borders in a liquid foam, are strongly deformed. As a result, the pores are also deformed, yielding a wide distribution of the pore diameters with a PDI of 9.6\%, as shown in Figure 5 (b). We assume that the presence of cross-links in the polymer network induces internal stresses responsible for the deformation of the Plateau borders. Note that in the non-cross-linked foams there are no chemical bonds between the chitosan chains and thus the chains have more degrees of freedom and can reorganize on a much smaller scale. As a consequence the structure of the resulting solid foams are more liquid-like than for the non-cross-linked freeze-dried foams. However, if non-crosslinked liquid foams lead to spherical pores with windows and cross-linked foams to wall-less structures, it could be interesting to freeze-dry foams with different cross-linking degrees and observe the influence of the cross-linking degree on the pore openness. We noted as well that, although cross-linked, the freeze-dried foams were not brittle and softer than the cross-linked foams which were dried via heating. However, compared to non-cross-linked freeze-dried foams, cross-linked freeze-dried foams were slightly harder. Mechanical tests could allow us later to understand the complex relationships between cross-linking, drying and the mechanical properties.

Interestingly, even though the cross-linked foams have the same composition and thus contain the same amount of material, freeze-drying leads to a three-dimensional material, while heating causes the foam to collapse under its own weight. We propose that the time at which the film breaks plays a role. In the case of drying via heating, cross-linking may be completed during the heating phase, but if the films break too early so that the critical modulus needed for the foam to support its own weight is not reached, the foam collapses. During freeze-drying, on the other hand, the foam is frozen so that its solvent, namely 1 vol \% acetic acid in water, may sublimate. Assuming that the pores open during freeze-drying, the structure is stabilized during this process by its frozen state, preventing the structure from collapsing. Looking at the Plateau borders, as shown in the inset of Figure 5 (a), one sees that their structure may result from the freeze-drying of a smooth polymer film. The Plateau borders are indeed thin and do not show any micropores characteristic of the sublimation of ice crystals. We thus argue that the polymer film was dense enough - or reorganized itself during freezing - to prevent the formation of ice crystals within the polymer matrix. 

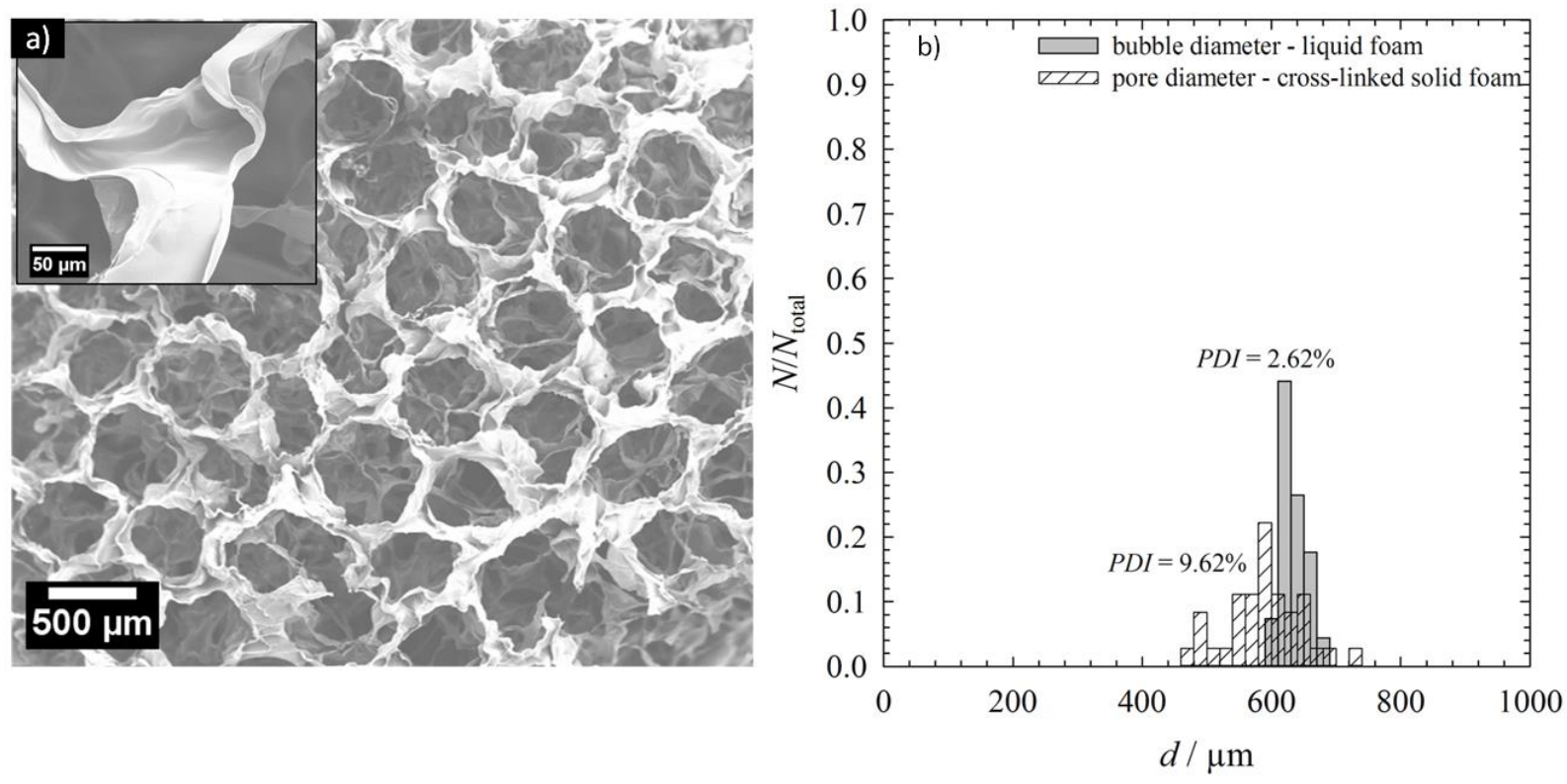

Figure 5: (a) Picture of a freeze-dried cross-linked foam. (b) Pore size distribution of the crosslinked freeze-dried foam and bubble size distribution of the corresponding liquid template. The average bubble diameter of the liquid template is $630 \pm 17 \mu \mathrm{m}$ and the average pore diameter is $591 \pm 57 \mu \mathrm{m}$.

\section{Conclusions}

Microfluidics is a useful tool to produce monodisperse foams with tuneable bubble sizes. We used this method to produce biobased scaffolds with high ordering. Interestingly, we managed to tune the structure and morphology of the scaffolds by modifying the solidification process of the templates. Immediate freeze-drying of the liquid templates leads to non-cross-linked scaffolds with spherical pores connected via windows. Letting the chitosan foam cross-link before freeze-drying one obtains scaffolds with even larger windows and deformed Plateau borders. We also investigated the possibility of drying the chitosan cross-linked foam as a simpler way than freeze-drying to produce a highly ordered scaffold. However, the resulting material collapsed under its own weight although monodispersity and ordering are retained after drying. Future research should investigate more precisely the structural changes of scaffolds freeze-dried at different stages of cross-linking in order to verify if a gradient of the size of the windows can be obtained. This could allow for a fine tuning of the windows sizes. Finally, the suitability of such scaffolds need to be tested as biomaterials for cellular growth and tissue engineering. 


\section{Acknowledgements}

We thank C. Honorez for his help in the design of the microfluidic chips. We also thank Dr. A. Fels for his support with SEM. We would like to thank as well Dr. B. Nestl, Dr. B. Nebel and S. Richter for the use of their freeze-drier. We acknowledge funding from the German Research Foundation (STU 287/4-1) and from the European Research Council (ERC, FP7/2007-2013, 307280-POMCAPS).

\section{References}

A. Barbetta, A. Gumiero, R. Pecci, R. Bedini, M. Dentini, Biomacromolecules 2009, 10(12), 3188-3192.

A. Barbetta, G. Rizzitelli, R. Bedini, R. Pecci, M. Dentini, Soft Matter 2010, 6(8), 1795-1792.

K.-Y. Chung, N. C. Mishra, C.-C. Wang, F.-H. Lin, K.-H. Lin, Biomicrofluidics 2009, 3, 022403.

M. Costantini, C. Colosi, J. Guzowski, A. Barbetta, J. Jaroszewicz, W. Święszkowski, M. Dentini, P. Garstecki, J. Mater. Chem. B 2004, 2(16), 2290-2300.

[5] D. Barby, Z. Haq, Unilever PLC, EP 0060138, 1982.

[6] C. Colosi, M. Costantini, A. Barbetta, R. Pecci, R. Bedini, M. Dentini, Langmuir 2013, 29(1), 82-91.

A. van der Net, A. Gryson, M. Ranft, F. Elias, C. Stubenrauch, W. Drenckhan, Colloids Surf., A 2009, 346(1-3), 5-10.

A. Testouri, C. Honorez, A. Barillec, D. Langevin, W. Drenckhan, Macromolecules 2010, 43(14), 6166-6173. Net, A. Lecchi, A. Salonen, E. Rio, R.-M. Guillermic, D. Langevin, W. Drenckhan, Colloids Surf., A 2012, 413, 17-24. chitosan, Pergamon Press: Oxford, UK, 1973. 
[11] J. Berger, M. Reist, J. M. Mayer, O. Felt, R. Gurny, Eur. J. Pharm. Biopharm. 2004, 67(1), 35-52.

A. Di Martino, M. Sittinger, M. K. Risbud, Biomaterials 2005, 26(30), 59835990.

[13] J. L. Drury, D. J. Mooney, Biomaterials 2003, 24(24), 4337-4351.

[14] M. F. Butler, Y.-F. Ng, P. D. A. Pudney, J. Polym. Sci. A 2003, 41(24), 39413953.

R. A. A. Muzzarelli, Carbohydr. Polym. 2009, 77(1), 1-9.

[16] J. Miras, S. Vílchez, C. Solans, J. Esquena, J. Colloid Interface Sci. 2013, 410, $33-42$.

A. van der Net, L. Blondel, A. Saugey, W. Drenckhan, Colloids Surf., A. 2007, 309, 159-176.

[18] W. Drenckhan, D. Langevin, Curr. Opin. Colloid Interface Sci. 2010, 15(5), 341-358.

[19] P. Marmottant, J.-P. Raven, Soft Matter 2009, 5(18), 3385-3388.

[20] E. Lorenceau, Y. Y. C. Sang, R. Höhler, S. Cohen-Addad, Phys. Fluids 2006, 18, 097103.

W. Drenckhan, S. Hutzler, Adv. Colloid Interface Sci. 2015, 224, 1-16.

[22]

S. Dimida, C. Demitri, V. M. De Benedictis, F. Scalera, F. Gervaso, A. Sannino, J. Appl. Polym. Sci. 2015, 132(28), 42256.

W. Kloek, T. von Vliet, M. Meinders, J. Colloid Interface Sci. 2001, 237(2), $158-166$.

J.-E. Park, J.-Y. Lee, H.-G. Kim, T.-R. Hahn, Y.-S. Paik, J. Agric. Food Chem. 2002, 50(22), 6511-6514.

[25] A. van der Net, W. Drenckhan, D. Weaire, S. Hutzler, Soft Matter 2006, 2(2), $129-134$. 


\section{Supplementary Information}

\section{Microfluidic Chips}
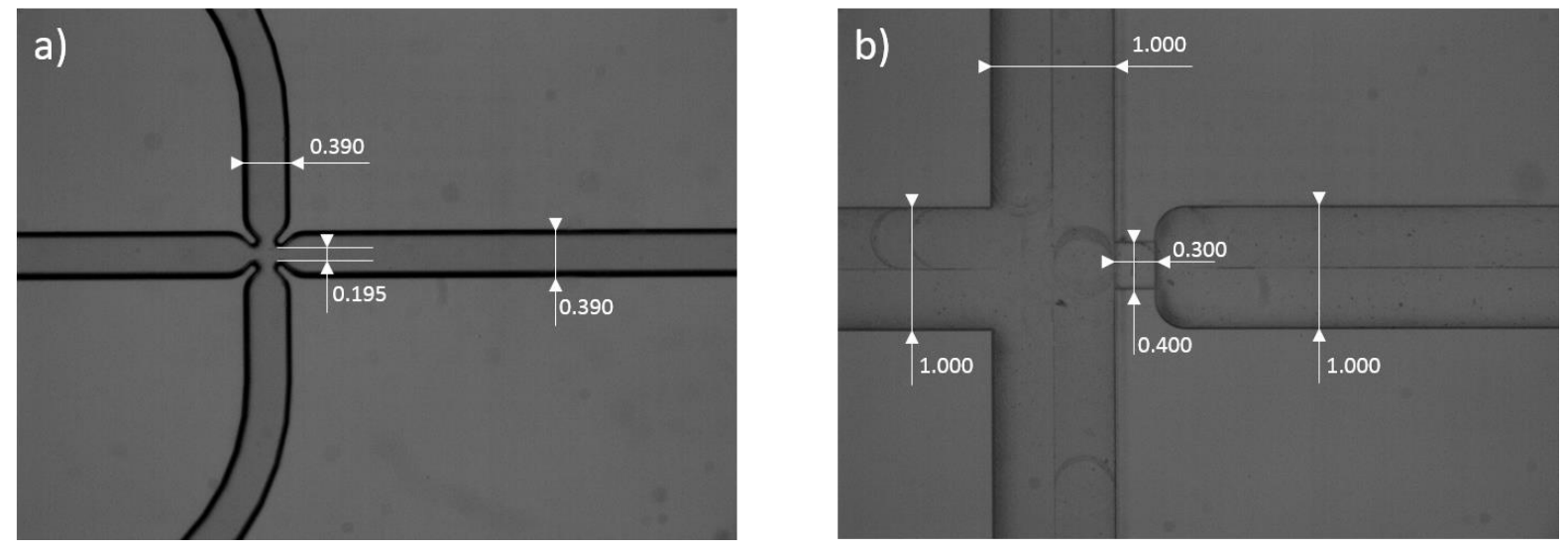

Figure S1: (a) Picture of the $190 \mu \mathrm{m}$ chip with the dimensions of the channels in $\mathrm{mm}$. The depth of the channels is $0.190 \mathrm{~mm}$. (b) Picture of the $400 \mu \mathrm{m}$ chip with the dimensions of the channels in $\mathrm{mm}$. The depth of the channels with a width of $1.000 \mathrm{~mm}$ is $0.800 \mathrm{~mm}$ while the depth of the constriction, which has a width of $0.400 \mathrm{~mm}$, is $0.400 \mathrm{~mm}$.

\section{Uncut Solid Foams}
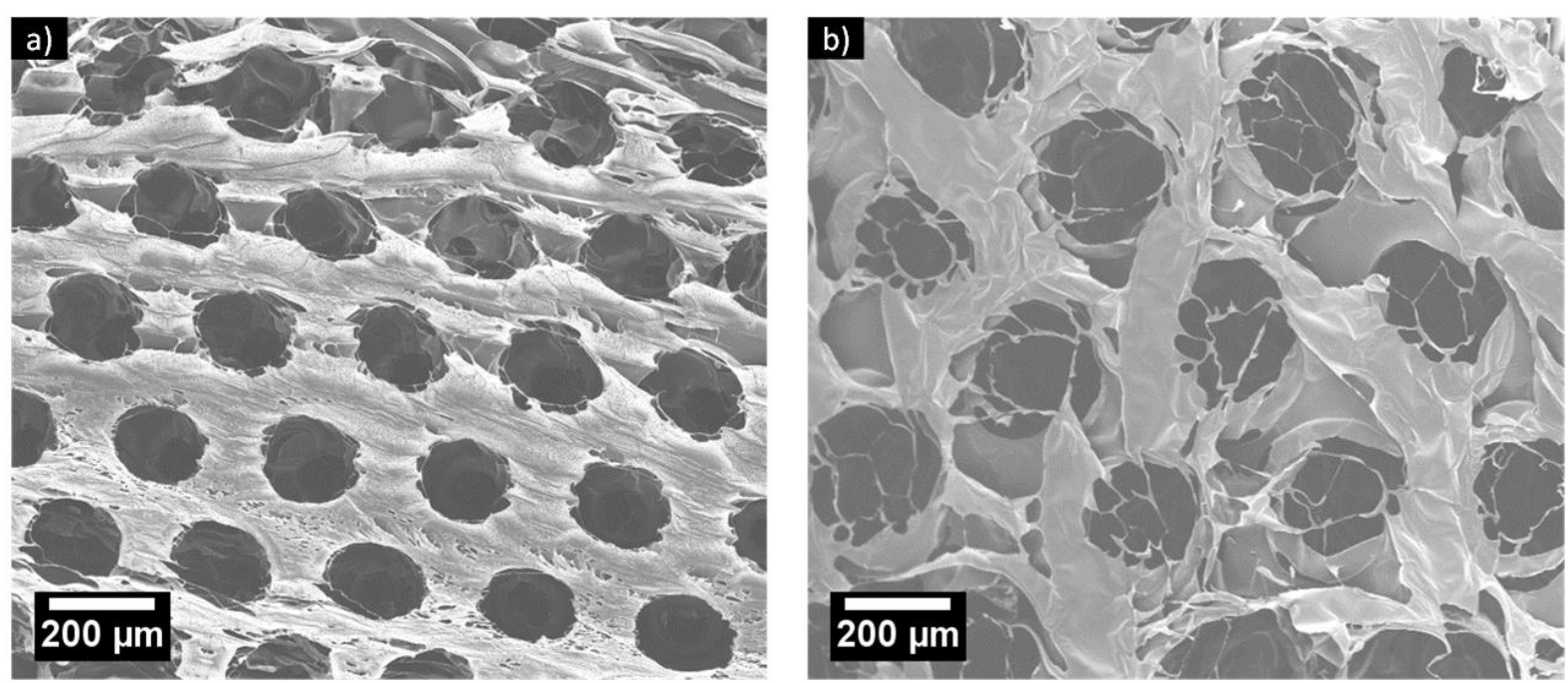

Figure S2: Pictures of the top layers of monodisperse non-cross-linked freeze-dried foams prepared with (a) the $190 \mu \mathrm{m}$ chip and (b) the $400 \mu \mathrm{m}$ chip.

\section{Oscillatory Rheometry}

The kinetics of cross-linking was measured with an oscillatory rheometer (Physica MCR 501 Rheometer from Anton Paar). The geometry used was cone-plate with the cone having a radius of $12.485 \mathrm{~mm}$ and an angle of $1.003^{\circ}$. The measurement position was $50 \mu \mathrm{m}$ and the 
measurements were performed for a $1 \%$ deformation at a frequency of $1 \mathrm{~Hz}$. The temperature was controlled with a Peltier system with a temperature accuracy of $0.1 \mathrm{~K}$. All gel point measurements were carried out at different temperatures for the same composition, that is 1.5 wt $\%$ chitosan cross-linked with 0.2 wt $\%$ genipin. All measurements were started 5 minutes after deposition of the sample in the rheometer.
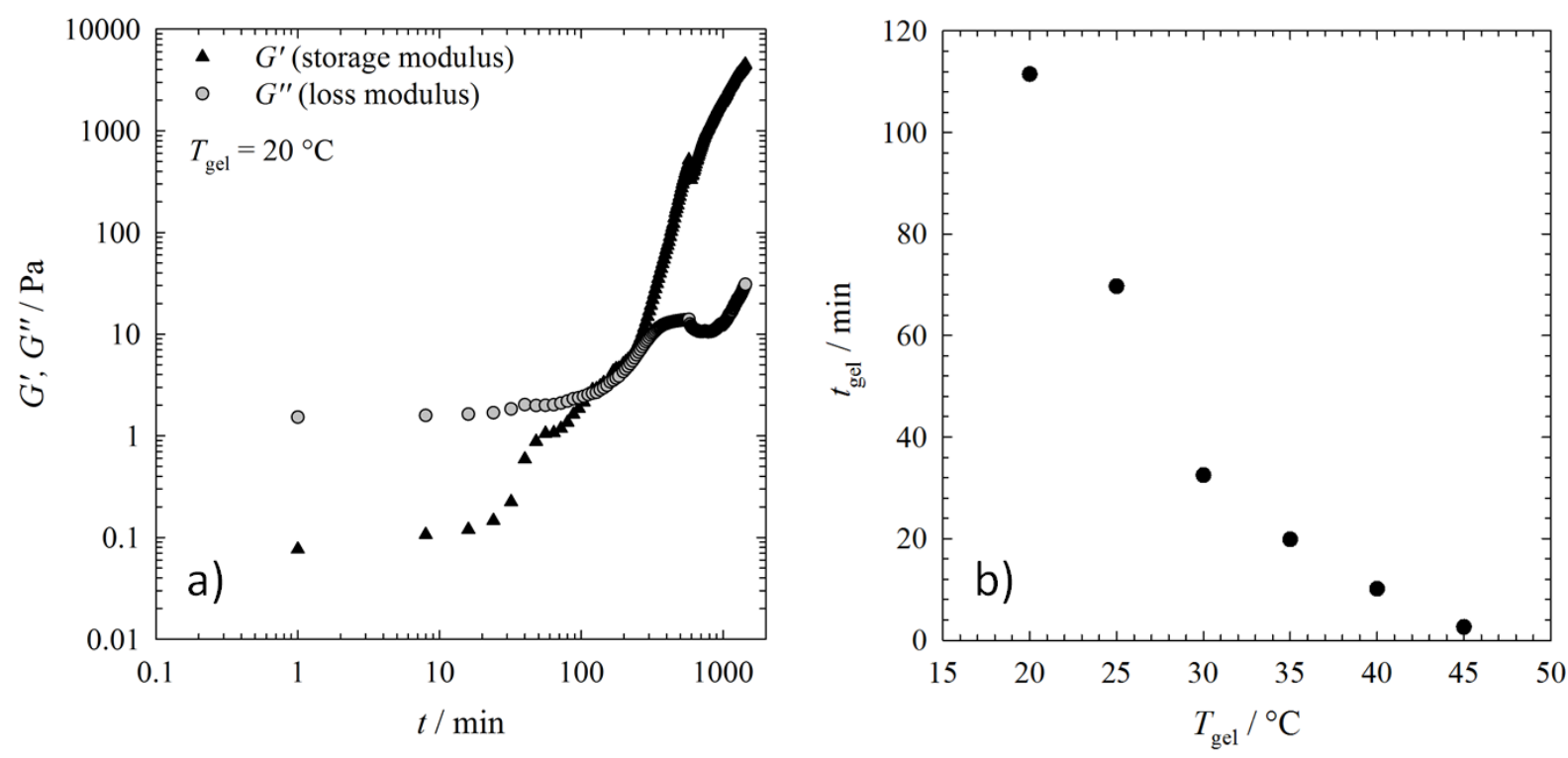

Figure S3: Gel points of the chitosan/genipin system at different temperatures. The inset shows the example of a gel point measurement at $20^{\circ} \mathrm{C}$ via oscillatory rheology. The gel point is defined as the intersect of the storage and loss moduli. 